\title{
Package 'saControlLimits'
}

May 12, 2015

Type Package

Title Computation of control limits using stochastic approximation.

Version 1.0

Date 2015-01-27

Author Capizzi Giovanna and Masarotto Guido.

Maintainer Masarotto Guido <guido.masarotto@unipd.it>.

Description The main function saControlLimits computes the control limits of a chart (single or combined) using the stochastic approximation algorithm described in Capizzi and Masarotto (2015). A simple parallel version of the same algorithm is also provided.

License Artistic-2.0

Imports $\operatorname{Rcpp}(>=0.11 .2)$

LinkingTo Rcpp

\section{R topics documented:}

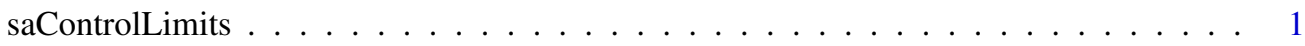

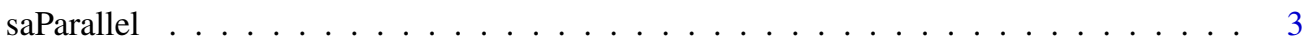

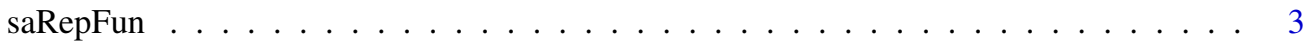

Index $\quad 5$

saControllimits Computation of control limits

\section{Description}

The function computes the control limits of a control charts using the stochastic approximation algorithm described in Capizzi and Masarotto (2015). The computation is distributed on multiple CPUs if a cluster has been previously created using saPStart.

\section{Usage}

saControllimits(hstart, Arl0, rlSim, ..., Nfixed $=500$, Afixed $=0.1$, Amin $=0.1$, Amax $=100$, delta $=0.1, q=0.55$, gamma $=0.01, \mathrm{Nmin}=1000, \mathrm{z}=3, \mathrm{Cmrl}=10$ ) 


\section{Arguments}

hstart A numeric vector containing the starting point;

Arlo The desired in-control average run-length;

rlSim A function used to simulate the run-length; see Details;

... Extra arguments for rlSim;

Nfixed, Afixed, Amin, Amax, delta, q, gamma, Nmin, z, Cmrl

Tuning constants; see Section 2 in Capizzi and Masarotto (2015).

\section{Details}

Function rlSim is used to simulate the run-length. It is called by saControllimits as

rlSim $(h=h, \ldots, \operatorname{maxrl}=\max r l, \operatorname{del} t a=d e l t a)$

where

- $\mathrm{h}$ : a numeric vector containing the control limits;

- ...: any other arguments needed to describe the control charts; for example the smoothing constant of an EWMA, the offset of a CUSUM, etc;

- maxrl: the maximum run-length, i.e., the function truncates the run-length simulation at $\operatorname{maxrl}$;

- delta: a numeric scalar used to estimate the stochastic approximation gain.

rlSim returns a vector (of the same length of $h$ ) containing the simulated run-lengths of all the combined control charts.

If delta is greater than zero, the return vector has, as attributes $r l+$ and $r l-, r l_{r i}^{+}$and $r l_{r i}^{-}$. Here, $r l_{r i}^{+}$and $r l_{r i}^{-}$denote the run-lengths obtained simulating from $h+\delta e_{i}$ and $h-\delta e_{i}$ where $e_{i}$ is the standard unit vector in the ith direction.

See the supplementary material of the paper for an example.

\section{Value}

A list with components

h A numeric vector containing the estimated control limits;

iter Number of iterations.

\section{Author(s)}

Capizzi Giovanna and Masarotto Guido.

\section{References}

Capizzi Giovanna and Masarotto Guido (2015) Efficient Control Chart Calibration by Simulated Stochastic Approximation, IIE Transanctions.

\section{See Also}

saPStart, saRepFun. 
saParallel

Computation of control limits using multiple cores

\section{Description}

saPStart, saPInit, saPSeed and saPStop are used to start, initialize, set the seed of the RNG streams and stop a cluster for saControlLimits and saRepFun.

\section{Usage}

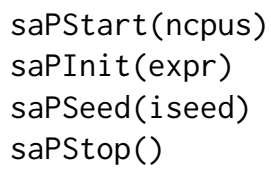

\section{Arguments}
ncpus
Number of cores to be used;
expr
An expression to evaluate on each cluster node;
iseed
An integer to be supplied to set.seed, or NULL not to set reproducible seeds.

\section{Note}

The cluster is created using the $\mathrm{R}$ standard package parallel-package.

\section{Author(s)}

Capizzi Giovanna and Masarotto Guido.

\section{See Also}

saControllimits, saRepFun.

\section{Description}

The function returns replicate $(n$, fun $(\ldots))$. The computation is distributed over multiple CPUs if a cluster has been previously created by saPStart.

\section{Usage}

$\operatorname{saRepFun}(n$, fun, ...)

\section{Arguments}

$\mathrm{n}$

fun

$\ldots$
Integer: the number of replications;

The function to be called;

Optional arguments to be passed to fun. 


\section{Author(s)}

Capizzi Giovanna and Masarotto Guido.

See Also

replicate, saPStart. 


\title{
Index
}

\author{
*Topic Parallel computation \\ saParallel, 3 \\ saRepFun, 3 \\ *Topic Statistical Process Monitoring \\ saControlLimits, 1 \\ replicate, 4 \\ saControllimits, 1, 3 \\ saParallel, 3 \\ saPInit (saParallel), 3 \\ saPSeed (saParallel), 3 \\ saPStart, 1, 2, 4 \\ saPStart (saParallel), 3 \\ saPStop (saParallel), 3 \\ saRepFun, 2, 3, 3
}

